EUROPEAN JOURNAL OF PURE AND APPLIED MATHEMATICS

Vol. 13, No. 1, 2020, 180-184

ISSN 1307-5543 - www.ejpam.com

Published by New York Business Global

\title{
Some applications of Ptolemy's theorem in secondary school mathematics
}

\author{
Samed J.Aliyev ${ }^{1, *}$, Shalala A.Hamidova ${ }^{1}$, Goncha Z.Abdullayeva ${ }^{1}$ \\ ${ }^{1}$ Department of Methods of Mathematics and its Teaching, Faculty of Mechanics and \\ Mathematics, Baku State University, Baku, Z.Khalilov str.23,, AZ1148, Azerbaijan
}

\begin{abstract}
We consider some applications of Ptolemy's theorem. In particular, we find a criterion for constructing an inscribed hexagon.
\end{abstract}

2020 Mathematics Subject Classifications: 97G10, 97G40

Key Words and Phrases: Ptolemy's theorem, inscribed rectangle, regular heptagon, inscribed hexagon.

Today, to improve the quality of teaching mathematics is one of biggest challenges faced in the field of education. To do so, you have to diversify the process of teaching, to improve the methods of teaching, to start using new approaches for some problems.

Since ancient times, the researchers have been studying the properties of inscribed polygons. The question of "Under what conditions is it possible to draw a polygon inside a circle? has always been of interest, and some criteria were found for inscribed polygons. The criterion for an inscribed rectangle provided in modern textbooks of secondary school geometry involves internal angles of rectangle. It says: for a rectangle to be inscribed in a circle, it is necessary and sufficient that the sum of its opposite angles be equal to $180^{\circ}$. These textbooks do not mention the relationships between the sides and diagonals of inscribed rectangle. Though, this is exactly what Ptolemy's famous theorem is about.

Not included in modern school textbooks, Ptolemy's theorem is a good criterion for constructing an inscribed rectangle which involves the sides and diagonals of a rectangle. It says: for a rectangle to be inscribed in a circle, it is necessary and sufficient that the sum of the products of its opposite sides be equal to the product of its diagonals.

In this work, we consider some problems which have been earlier solved by traditional methods, and we easily solve these problems by using new approaches, namely, Ptolemy's theorem. The results obtained reveal the effectiveness of this new approach. It incites students attention to the subjects studied during geometry classes and encourages them

*Corresponding author.

DOI: https://doi.org/10.29020/nybg.ejpam.v13i1.3605

Email addresses: samed59@bk.ru (S. J.Aliyev),

shalalahamidova@hotmail.com (S. A.Hamidova), a.q.z.41@mail.ru (G. Z.Abdullayeva) 
to learn more about these subjects. As a result, students get more interested in geometry and start loving it.

Inscribed polygons have been studied in [1], [2], [5]. Also, the criteria for constructing some inscribed polygons have been found in [3], [4].

In this work, we consider some applications of Ptolemy's theorem. Namely, using Ptolemy's theorem,

1) we prove some property of a point lying on a circle circumscribed about some regular triangle;

2) we prove some property of a regular heptagon;

3) we find a criterion for constructing an inscribed hexagon.

We first prove that if the point $M$ lies on a circle circumscribed about the regular triangle $A B C$, then one of the intervals $M A, M B, M C$ is equal to the sum of two other intervals.

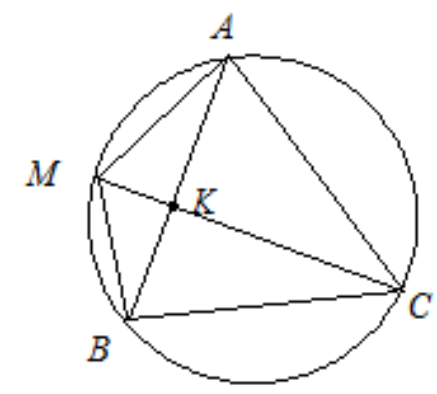

Figure 1: Circle circumscribed about the regular triangle $A B C$.

Let the point $M$ lie on the $\operatorname{arc} A B$ (Figure 1). Let's prove that

$$
M A+M B=M C .
$$

We will use traditional methods. As $\angle B M C=\angle C M A$, due to angle bisector property of a triangle we have

or

$$
\frac{M B}{M A}=\frac{B K}{K A}
$$

$$
B K \cdot M A=M B \cdot K A,
$$

Regarding similar triangles $B M C$ and $K M A$, we have

$$
\frac{B C}{K A}=\frac{M C}{M A}
$$

or

$$
B C \cdot M A=M C \cdot K A,
$$

As $B C=B K+K A$, from the last equality, using (2), we get the validity of (1):

$$
(B K+K A) \cdot M A=M C \cdot K A
$$




$$
\begin{aligned}
& B K \cdot M A+M A \cdot K A=M C \cdot K A, \\
& M B \cdot K A+M A \cdot K A=M C \cdot K A,
\end{aligned}
$$

or

$$
M B+M A=M C .
$$

But, this relation can be obtained quite easily without using bisector property and similar triangles, just by means of Ptolemy's theorem.

As the rectangle $M A B C$ is inscribed, by Ptolemy's theorem we have

$$
M A \cdot B C+M B \cdot A C=M C \cdot A B .
$$

Considering the conditions $B C=A C=A B$, we get the validity of (1).

Now let's prove that if the points $A, B, C, D$ are the consecutive vertices of a regular heptagon (Figure 2), then the following relation holds:

$$
\frac{1}{A B}=\frac{1}{A C}+\frac{1}{A D}
$$

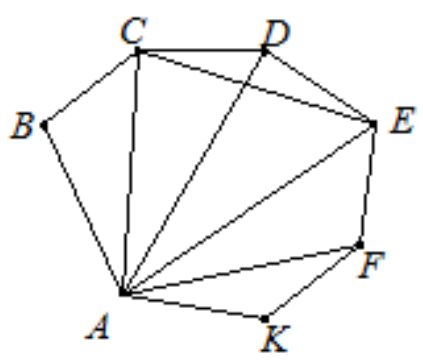

Figure 2: Regular heptaqon $A B C D E F K$.

Let the point $E$ be a vertex of a regular heptagon next to $D$. The triangles $A B C$ and $A C D$ are equal to the triangles $C D E$ and $A F E$, respectively. Hence $A C=C E$ and $A D=A E$. Taking into account the equality of these intervals and the conditions $D E=C D=A B$, by Ptolemy's theorem applied to the rectangle $A C D E$ we get the validity of $(3)$ :

$$
\begin{gathered}
A C \cdot D E+A E \cdot C D=C E \cdot A D, \\
A C \cdot A B+A D \cdot A B=A C \cdot A D, \\
\frac{1}{A B}=\frac{1}{A C}+\frac{1}{A D} .
\end{gathered}
$$

Finally, let's find a criterion for constructing an inscribed hexagon, which involves its sides and large diagonals, using Ptolemy's theorem. In other words, let's prove that the product of large diagonals of an inscribed hexagon is equal to the sum of products of its 

consecutive non-adjacent sides and products of its opposite sides with a large diagonal which does not intersect any of these sides.

Let the hexagon $A B C A_{1} B_{1} C_{1}$ be inscribed in a circle (Figure 3 ).

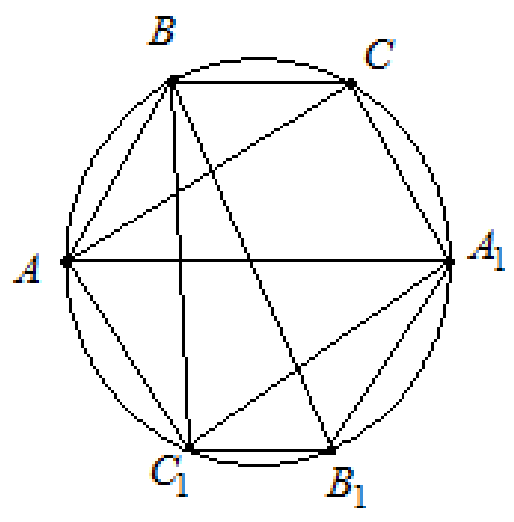

Figure 3: The hexagon $A B C A_{1} B_{1} C_{1}$ be inscribed in a circle.

We must prove that the following relation is true in the hexagon $A B C A_{1} B_{1} C_{1}$ :

$$
\begin{aligned}
& A A_{1} \cdot B B_{1} \cdot C C_{1}=A B \cdot C A_{1} \cdot B_{1} C_{1}+B C \cdot A_{1} B_{1} \cdot A C_{1}+ \\
& +A B \cdot A_{1} B_{1} \cdot C C_{1}+B C \cdot B_{1} C_{1} \cdot A A_{1}+C A_{1} \cdot A C_{1} \cdot B B_{1} .
\end{aligned}
$$

Applying Ptolemy's theorem to the rectangles $A C A_{1} C_{1}, A_{1} B_{1} C_{1} B, A B C C_{1}, A B C A_{1}$, respectively, we obtain

$$
\begin{gathered}
A A_{1} \cdot C C_{1}=A C \cdot A_{1} C_{1}+A C_{1} \cdot C A_{1}, \\
A_{1} C_{1} \cdot B B_{1}=B A_{1} \cdot B C_{1}+A_{1} B_{1} \cdot B C_{1}, \\
A C \cdot B C_{1}=A B \cdot C C_{1}+B C \cdot A C_{1}, \\
A C \cdot B A_{1}=A B \cdot C A_{1}+B C \cdot A A_{1} .
\end{gathered}
$$

Then, multiplying (5), (6), (7), (8) by $B B_{1}, A C, A_{1} B_{1}, B_{1} C_{1}$, respectively, and adding up the resulting equalities we obtain the relation (4).

The equality (4) is a relationship between the sides and diagonals of an inscribed hexagon. That's why it can well be called an analogue of Ptolemy's theorem for an inscribed hexagon. 


\section{References}

[1] V.A. Dalinger; Graphics teaches how to think , Matematika v shkole, (1990), No4, p.32-36. (in Russian)

[2] V.Y.Kutsenok; The circle helps to solve problems ,Matematika v shkole,(1990), No2, p.55-30. (in Russian)

[3] V.Y.Kutsenok; Teaching the methods of solving geometric problems based on the use of auxiliary circle, $\mathrm{PhD}$ thesis in pedagogics, Moscow, (1992).(in Russian)

[4] Y.P.Ponarin; Elementary geometry, v.1. Moscow, MTsNMO, (2008), 312 p. (in Russian)

[5] I.F.Sharigin; Geometry, M.: Drofa, (1999), 352 p. (in Russian) 